\title{
Vasodilator stress with adenosine and the gender preponderance for tolerability and manifestation of adverse symptoms: Is there a physiological basis?
}

Pharmacological stress testing with adenosine and adenosine analogs has been increasingly utilized for stress perfusion imaging over the last decade and now constitutes nearly half of all nuclear cardiology stress testings across the world. The commonly encountered side effects in association with adenosine injection include flushing, chest discomfort, throat, neck or jaw discomfort, abdominal pain, lightheadedness and dizziness, symptomatic hypotension. Two modifications of adenosine stress protocols have evolved in the clinical practice for reducing the adverse effects of adenosine infusion: (a) combination of adenosine infusion with simultaneous low-level exercise ${ }^{1-3}$ significantly reduces adverse effects of adenosine infusion. In addition, image quality is bettered by increasing heart-to-liver or heartto-gut radiotracer uptake; the same has been reported with the newer stress agent regadenoson ${ }^{3}$ (b) reduction in duration of adenosine infusion by 1-2 minutes reduces the duration of adverse effects.

Another observation, which is relatively less highlighted in the literature but an observation that is quite frequent with the practitioners is higher incidence of the symptoms in female population compared to the male counterparts undergoing the vasodilator stress with adenosine. One of the early reports by Thomas et al. ${ }^{1}$ had made a mention of this in their results $(5.7 \%$ vs $1.8 \%)$, though this has been largely unreported in subsequent years. This gender preponderance of adenosine-related symptoms during stress testing is a common observation in routine setting: a male patient who can be subjected to appropriate low-level exercise can undergo the entire adenosine stress test without any symptom. The reason for such discrepancy in the incidence is also unexplained at present. Also, there has been no concrete data at present on whether the approaches to reduce side effects such as combination with low-level exercise or shorter infusion

J Nucl Cardiol 2015;22:1158

$1071-3581 / \$ 34.00$

Copyright (c) 2015 American Society of Nuclear Cardiology. duration equally effective in men vs. women. However, the latter appears more effective in women compared to men due to the higher incidence of symptoms in them. Interestingly, such observation is also being noticed with the newer A-2a specific stressor such as regadenoson ${ }^{4,5}$ ( $80 \%$ in females vs $70 \%$ in males); however, the overall tolerability was better and the incidence of symptoms was less compared to adenosine. ${ }^{5}$

\section{Conflict of interest None.}

Sandip Basu, Radiation Medicine Centre, Bhabha Atomic Research Centre (BARC), Tata Memorial Hospital, Annexe Building, Jerbai Wadia Road, Parel, Mumbai 400 012, Maharashtra, India; drsanb@yahoo.com.

\section{References}

1. Thomas GS, Prill NV, Majmundar H, Fabrizi RR, Thomas JJ, Hayashida C, et al. Treadmill exercise during adenosine infusion is safe, results in fewer adverse reactions, and improves myocardial perfusion image quality. J Nucl Cardiol 2000;7:439-46.

2. Samady H, Wackers FJ, Joska TM, Zaret BL, Jain D. Pharmacologic stress perfusion imaging with adenosine: role of simultaneous low-level treadmill exercise. J Nucl Cardiol 2002;9:188-96.

3. Baskot B, Obradovic S, Rafajlovski S, Gligic B, Orozovic V, Ratkovic N, et al. Adenosine stress protocols for nuclear cardiology imaging. Prilozi 2008;29:281-9.

4. Thomas GS, Thompson RC, Miyamoto MI, Ip TK, Rice DL, Milikien D, et al. The RegEx trial: a randomized, double-blind, placebo- and active-controlled pilot study combining regadenoson, a selective $\mathrm{A}(2 \mathrm{~A})$ adenosine agonist, with low-level exercise, in patients undergoing myocardial perfusion imaging. J Nucl Cardiol 2009;16(1):63-72.

5. Cerqueira MD, Nguyen $P$, Staehr $P$, Underwood SR, Iskandrian AE, ADVANCE-MPI Trial Investigators. Effects of age, gender, obesity, and diabetes on the efficacy and safety of the selective A2A agonist regadenoson versus adenosine in myocardial perfusion imaging integrated ADVANCE-MPI trial results. JACC Cardiovasc Imaging 2008;1(3):307-16.

doi:10.1007/s12350-015-0215-3 